\title{
Evaluation of the effectiveness of decontamination of dental syringes
}

\author{
K. Vickery, ${ }^{1}$ A. Pajkos, ${ }^{2}$ and Y. Cossart, ${ }^{3}$
}

\begin{abstract}
Aim Steam autoclaving is the gold standard for decontaminating dental instruments, but worldwide disinfection is still widely employed. We have evaluated a range of procedures for their ability to inactivate duck hepatitis B virus contaminating dental syringes.

Methods Residual infectivity of virus suspensions following 2\% glutaraldehyde treatment, ultrasonication or steam sterilisation at $121^{\circ}$ or $134^{\circ}$ was assayed by injecting day-old ducklings and examining their livers for viral DNA 2.5 weeks later. Dental syringes were contaminated with DHBV positive blood, then treated by the same methods. An anaesthetic cartridge containing water was loaded into the syringe and $400 \mu \mathrm{l}$ aliquots used to inject day-old ducklings. Used dental syringes were examined by Scanning Electron Microscopy.

Results Suspension test:- ultrasonic treatment failed to inactivate DHBV in suspension, but complete inactivation was achieved by $2 \%$ glutaraldehyde and autoclaving. Syringe test:neither ultrasonic treatment nor glutaraldehyde inactivated DHBV. Autoclaving at $134^{\circ}$ ( 3 minutes) permitted transmission to $1 / 16$ ducklings but steam sterilisation at $121^{\circ}$ ( 15 minutes $)$ was effective. Electronmicroscopy demonstrated organic debris (biofilm) in the lumen of used syringes.

Conclusion Short autoclaving cycles, albeit at raised temperatures, may fail to inactivate the virus because of poor steam penetration, inadequate heat transfer and the accumulation of protective biofilm.
\end{abstract}

Tnfection control practices have come under increasing public 1 scrutiny over the last decade in the light of significant medical problems associated with the acquisition of blood borne pathogens following both medical ${ }^{1}$ and dental procedures. ${ }^{2}$

Successive revisions of official guidelines have made progressively more stringent recommendations for decontamination of instruments in office practice, to bring them into line with current 'best practice' procedures in hospitals. The National Health and Medical Research Council of Australia (NHMRC) ${ }^{3}$ recommends that all instruments, materials and medications introduced into sterile tissue must be sterile. For instruments and equipment, this should be achieved by using single-use items only, or, if the items are designed for multi-use, they must be scrupulously cleaned and adequately sterilised.

The level of risk for invasive surgical, including dental procedures

\footnotetext{
${ }^{*}$ Post Doctoral Fellow, ${ }^{2}$ Phd Student,${ }^{3}$ Bosch Professor of Infectious Diseases, The Australian Centre for Hepatitis Virology and the Department of Infectious Diseases, University of Sydney, Sydney, Australia. 2006

${ }^{*}$ Correspondence to: $K$. Vickery

REFEREED PAPER

Received 14.06.99; Accepted 29.11.99

(C) British Dental Journal 2000; 189: 620-624
}

is ranked by Spaulding's ${ }^{4}$ classification scheme as 'critical' and therefore all instruments used must be sterile. Items that come in contact with intact mucous membranes however are classified as semi-critical and must be subjected to high-level disinfection with an approved disinfectant such as glutaraldehyde. In contrast to Spaulding's classification The British Dental Association (BDA) Advisory Service ${ }^{5}$ recommends sterilisation of all instruments that become contaminated with oral and other body fluids. Sterility can be achieved by: autoclaving (steam sterilisation under pressure at $121^{\circ}$ to $134^{\circ} \mathrm{C}$ ), or by dry heat at $160^{\circ} \mathrm{C}$. Large scale systems of radiation, ethylene-oxide, or chemical treatment are also effective. Autoclaving is generally accepted as the method of choice to render contaminated instruments safe for reuse. ${ }^{6}$ Currently steam sterilisation using portable autoclaves is recommended for sterilising dental instruments for use in critical sites but it has been reported that some practitioners are still utilising high-grade disinfection such as buffered alkaline glutaraldehyde ${ }^{7}$ although this is now recommended for use in semi-critical areas only. This lack of compliance may arise because there is only limited direct scientific evidence about the effectiveness of various disinfection/sterilisation methods in the dental practice.

Blood borne viruses are of major concern in the health care setting. Hepatitis B virus (HBV) is the most resistant blood borne virus in the environment and can persist for extended periods on contaminated surfaces or under the fingernails of providers. ${ }^{8,9}$ It is also present in high concentration in the blood and saliva and currently there are many more carriers of $\mathrm{HBV}$ in the community than there are carriers of HIV. Therefore, the effective elimination of $\mathrm{HBV}$ is the most critical indicator for assessing efficacy of infection control procedures.

Unfortunately the lack of suitable infectivity models for HBV, such as tissue culture or animal inoculation, makes assessment of disinfectant efficacy difficult. The polymerase chain reaction (PCR) is a sensitive and specific test for the detection of viral nucleic acids and has been successfully employed to trace the fate of viruses in the environment. ${ }^{10}$ However, since nucleic acids survive treatments with heat, solvents and fixatives known to destroy microbial infectivity, PCR results obtained from disinfected or sterilised instruments may be misleading. ${ }^{11}$

Resistance to chemical and physical agents is very similar for members of each virus family, and this similarity is very useful when selecting test organisms for evaluation of disinfection and sterilisation procedures. Studies of the duck hepatitis B virus (DHBV) in particular have revealed similar biological and structural characteristics as HBV. The DHBV model also appears to have similar disinfectant inactivation kinetics to those reported in the very limited chimpanzee transmission studies of HBV. ${ }^{12,13}$ DHBV reaches high titre $>10^{8} \mathrm{ID}_{50} / \mathrm{ml}\left(10^{8}\right.$ times the amount of virus required to infect half of the experimental animals is present in each $\mathrm{ml}$ of blood) in the blood of infected ducks, and day-old ducklings are exquisitely sensitive to infection. These advantages have led to adoption of 


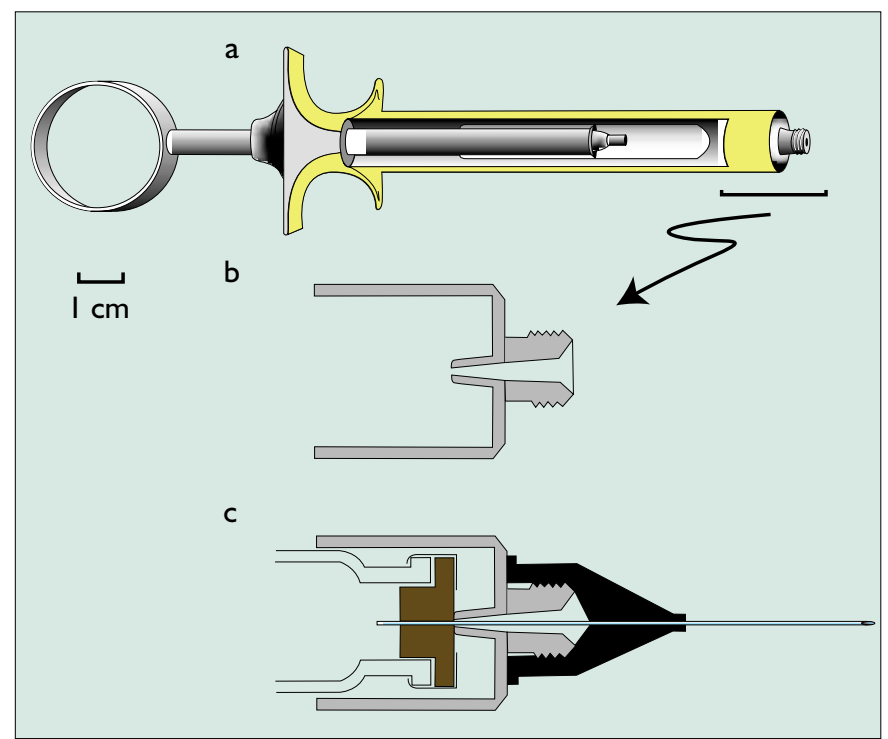

Fig. I $a$, Diagrammatic representation of a dental syringe $b$, enlargement of the cannula region and $c$, relationship between anaesthetic cartilage, dental needle and the cannula of the syringe.
2 Ultrasonication in UltraDose Ultrasonic Cleaning Solution (L\&R Manufacturing Company, New Jersey, USA) was mixed with an equal volume of blood/PBS mixture. This detergent blood mixture was placed into an ultrasonic bath (Bio-Sonic UC 300 Whaledent) for 10 minutes at an operating frequency of $40 \mathrm{kHz}$ $(n=8)$. The ultrasonic bath was set up by an experienced practitioner and its operation validated by the foil test.

3 The blood/PBS mixture was subjected to autoclave sterilisation at $134^{\circ} \mathrm{C}$ at $200 \mathrm{KPa}$ for 3 minutes holding time, drying time 20 minutes in a Tuttnaur 2540 EKA autoclave (Tuttnauer, Jerusalem, Israel) $(\mathrm{n}=8)$.

4 The blood/PBS mixture was subjected to autoclave sterilisation at $121^{\circ} \mathrm{C}$ at $103.4 \mathrm{Kpa}$, for 15 minutes, with a 20 minute drying cycle in an Atherton Cyclomatic Control Hospital Autoclave (Athertons, Sydney, Australia) $(\mathrm{n}=8)$.

The test samples and the positive control were then diluted 1/500 which served to minimised any disinfectant toxicity to the ducks and to prevent further disinfectant activity. Residual viral activity was determined by inoculation of $1 \mathrm{ml}$ of the diluted mixture into the peritoneal cavity of groups of one-day-old ducks as shown in Table 1.

The ducks were euthanased at 2.5 weeks of age and their livers removed for DHBV DNA analysis as described by Deva. ${ }^{11}$

DHBV as a model for disinfectant and antiviral testing by the worlds regulatory bodies. ${ }^{14}$

Education of the dental health care professionals about the parameters that influence disinfectant efficacy is of paramount importance in gaining informed compliance with regulatory guidelines. It is also important that the guidelines are built on firm scientific evidence that takes into account the multiple parameters such as shape of instrument, amount and type of biological contaminant which influence efficacy in the field as opposed to results obtained using artificial test protocols. With this in mind we have used DHBV to determine inactivation efficacy of autoclaving and glutaraldehyde disinfection of blood soiled dental syringes.

\section{Methods}

Experimental animals

Pekin Aylesbury crossbred ducks were obtained from a commercial supplier. The University of Sydney Animal Ethics Committee approved all experimental work. Ducklings were obtained from DHBV negative flocks and serum samples taken before inoculation were shown to be DHBV DNA free by dot-blot hybridization.

\section{DHBV positive source animals}

The prototype Australian strain of $\mathrm{DHBV}^{15}$ was used to infect five one-day-old ducklings. These animals were used as the source biohazardous ducks during peak viraemia (from 14 to 21 days of age). The viral titre of source animals was calculated to be $10^{7} \mathrm{ID}_{50}$ doses/ml from the amount of DHBV DNA detected by dot-blot hybridisation.

\section{Experimental protocol}

\section{Experiment 1. In vitro testing of decontamination procedures}

Fresh blood from a DHBV positive source duck was collected into heparin (Delta West, Bently, Western Australia) $(10 \mathrm{IU} / \mathrm{ml})$ prior to mixing with an equal volume of PBS. The blood/PBS mixture $(500 \mu l)$ was then mixed with an equal volume of test mixtures or phosphate buffered saline (PBS) for the positive control. The following procedures were tested, the number of ducks per group is in parentheses:

$12 \%$ glutaraldehyde (Aidal Plus, Whiteley Industries Pty Ltd, Sydney, Australia). The disinfectant and the blood were allowed to react at room temperature for $1(n=7)$ and 5 minutes $(n=8)$.
Table I Assessment of the efficiency of various disinfectant/sterilisation procedures against DHBV in the presence of blood/PBS mixture.

\begin{tabular}{lcc}
\hline Disinfectant & $\begin{array}{c}\text { Contact/sterilisation } \\
\text { time (min) }\end{array}$ & $\begin{array}{c}\text { DHBV positive/total } \\
\text { number of ducks }\end{array}$ \\
\hline Blood/PBS (Control) & 5 & $8 / 8$ \\
Glutaralderhyde 2\% & 1 & $0 / 8$ \\
Glutaralderhyde 2\% & 5 & $0 / 7$ \\
UltraDose & 10 & $8 / 8$ \\
Autoclave $134^{\circ} \mathrm{C}$ & 3 & $0 / 8$ \\
Autoclave $121^{\circ} \mathrm{C}$ & 15 & $0 / 8$ \\
\hline
\end{tabular}

Experiment 2. Effect of instrument use

Auto aspirating cartridge dental syringes (Astra Pharmaceuticals Pty Ltd, Nth Ryde, Australia) were used. Figure 1 is a diagrammatic representation of a dental syringe showing the relationship between the anaesthetic cartridge, new dental needle and the cannula of the syringe. The width of the cannula that the dental needle had to tra-

Prior to each experiment fresh blood was obtained from a DHBV positive source duck. The patient end of the dental syringes were dipped into a 50:50 mixture of DHBV positive duck blood and PBS for 3 minutes to simulate blood contaminated saliva. In order to ensure complete contamination of the inner surfaces, blood seepage through the lumen was observed. Upon removal, the instruments were hung vertically and allowed to dry for 5 minutes. The syringes were then subjected to one of the following treatments:

1 No treatment - Positive control $(n=8)$. Dried blood:PBS mix was not removed from either the internal or external surfaces.

2 Water wash and scrub $(n=8)$. Visible blood was removed by brushing under tap water.

3 Ultrasonication $(n=8)$. Visible blood was removed by brushing under tap water. The syringes were then placed into a Bio-Sonic UC 300 ultrasonic bath containing UltraDose Ultrasonic Cleaning Solution for 10 minutes at an operating frequency of $40 \mathrm{kHz}$. At the end of the treatment syringes were rinsed thoroughly under running tap water.

4 Glutaraldehyde treatment ( $n=8)$. Visible blood was removed by brushing under tap water. Syringes were then soaked in $2 \%$ glutaraldehyde (Whiteley Industries Pty Ltd, Australia) for 20 verse to puncture the anaesthetic cartridge varied between 1-2 mm. 


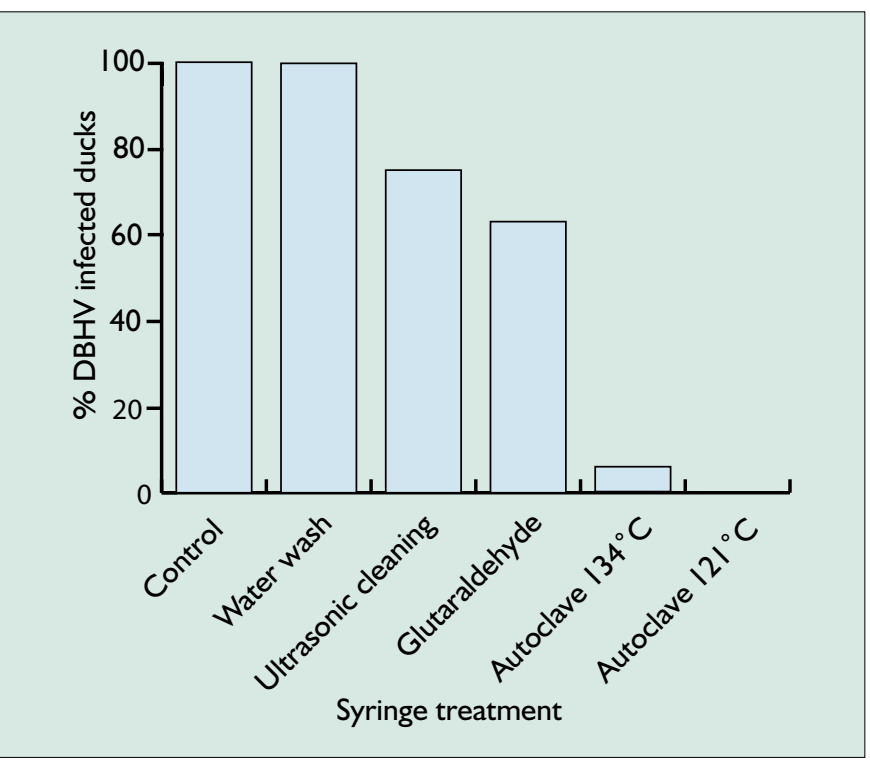

Fig. 2 Percentage transmission of DHBV infection following various decontamination procedures of dental syringes as observed in recipient animals.

minutes prior to thorough rinsing in tap water.

5 Autoclaving at $134^{\circ} \mathrm{C}$ at $200 \mathrm{KPa}$ for 3 minutes holding time, drying time 20 minutes in a Tuttnaur 2540 EKA downward displacement autoclave $(n=16)$. Visible blood was removed by brushing under tap water and dried.

a) Eight syringes were autoclaved bagged in Stericlin sleeves.

b) Eight syringes were autoclaved unwrapped.

A DUOR FLASH (Propper, New York) chemical indicator strip was included in each cycle to monitor physical parameters. The steriliser was equipped with a print-out facility and the recorded physical parameters after each cycle confirmed the efficient operation of the autoclave.

6 Autoclaving at $121^{\circ} \mathrm{C}$ at $103.4 \mathrm{Kpa}$, for 15 minutes, with a 20 minute drying cycle in an Atherton Cyclomatic Control Hospital Autoclave $(n=24)$. Dried visible blood was removed from the dental syringes by brushing under tap water and the syringes dried.

a) Sixteen syringes were autoclaved bagged in Stericlin sleeves

b) Eight syringes were autoclaved unwrapped.

A chemical indicator strip was included in each cycle to monitor physical parameters. The pressure and temperature gauges were monitored during each autoclave cycle.

All visible blood was removed from all the treated syringes (groups 2-6). None of the syringe cannulas were blocked and all appeared to be visually clean.

At the completion of each treatment session a $1.8 \mathrm{ml} \mathrm{dH_{2 }} \mathrm{O}$ filled anaesthetic cartridge was loaded into each dental syringe and a new sterile $27 \mathrm{G} \leftrightarrow 7 / 8^{\prime \prime}$ Terumo Dental needle attached in the normal fashion with no deliberate attempt being made to scrape the biofilm coating the inner surface. The contents of the cartridge were expelled into a sterile tube and a $400 \mu \mathrm{L}$ aliquot injected into the peritoneal cavity of a one-day-old duck.

The ducks were euthanased at 2.5 weeks of age and their livers removed for DHBV DNA analysis. ${ }^{11}$

\section{Detection of DHBV DNA by dot blot analysis}

Serum A $25 \mu \mathrm{L}$ aliquot of serum was denatured by the addition of $25 \mu \mathrm{L} 1 \mathrm{M} \mathrm{NaOH}$ and spotted onto Genescreen nylon membrane (Dupont, Boston, USA) using a biodot apparatus (Bio-Rad Laboratories, Richmond, CA, USA). Membranes were then hybridised with $32^{\mathrm{P}}$ labelled DHBV DNA, washed and autoradiographed as previously described. ${ }^{15}$

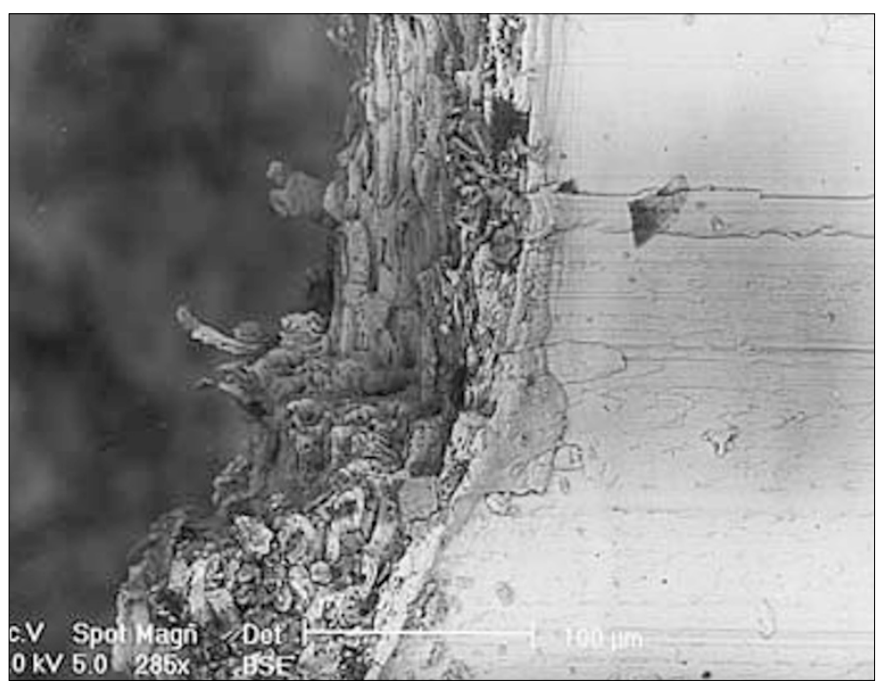

Figure 3. Scanning electron microscopy (Back Scattered Electron image) through the cannula of a dental syringe used in routine dental practice showing extensive biofilm formation. Measuring bar represents $100 \mu \mathrm{m}$

Liver Liver samples taken at the time of euthanasia $\left(0.2 \mathrm{~cm}^{3}\right)$ were placed in digestion buffer containing: $50 \mathrm{mM}$ Tris/ $\mathrm{HCl} \mathrm{pH} \mathrm{7.5,} 150$ $\mathrm{mM} \mathrm{NaCl}, 2 \mathrm{mM}$ EDTA, 1\% SDS and proteinase $\mathrm{K}(150 \mu \mathrm{g} / \mathrm{ml})$. Samples were incubated overnight at $37^{\circ} \mathrm{C}$ and then extracted using a standard phenol: chloroform method and then ethanol precipitated. Extracted DNA was then spotted onto nylon membrane and hybridised as described above.

Experiment 3. Scanning Electron Microscopy (SEM) of used dental syringes

Five dental syringes used in practice were obtained from dental practitioners to determine the extent to which the cannula becomes coated with organic material during routine use. The dental syringe was carefully cut in half without the use of lubricants to expose the inner surface of the cannula.

The samples were mounted on specimen studs with conductive carbon paint. They were examined in a SEM (XL-30 CP Philips) with a spot size 5 and accelerating voltage of 20 using scanning electrons and back scattered electrons.

\section{Results}

All ducklings were shown to be DHBV negative prior to both experiments.

Experiment 1. In vitro testing of decontamination procedures All the control ducks were positive for DHBV in their livers at 2 weeks of age. Under the conditions of the test, ultrasonication with UltraDose failed to inactivate DHBV while complete inactivation was achieved following both methods of autoclaving or glutaraldehyde treatment (see Table 1).

Experiment 2. Effect of instrument use

Two of the group 2 (water wash) ducks died due to unrelated causes and were excluded from further analysis.

All the ducks (8/8) in the control group that received samples from unwashed syringes became DHBV positive. Manual removal of visible dried blood by brushing under water failed to decrease the transmission of DHBV with all the ducks becoming DHBV positive (6/6). Ultrasonic cleaning of the contaminated syringes reduced the transmission rate to $75 \%$ (6/8). Manual cleaning followed by glutaraldehyde disinfection reduced the infection rate further to $62.5 \%$ $(5 / 8)$. After autoclaving at $134^{\circ} \mathrm{C}$ for 3 minutes one duck out of 16 
(6.25\%) became DHBV positive. The duck was infected from a syringe that was unbagged for autoclaving (see Fig 2). However, autoclaving using the longer 15 minute cycle at the lower temperature of $121^{\circ} \mathrm{C}$ completely inactivated DHBV $(\mathrm{n}=24)$.

\section{Experiment 3. Scanning electron microscopy of used dental syringes}

Examination of dental syringes used in routine dental practice with either scanning electrons or back scattered electrons revealed extensive contamination of the cannula with organic debris. The depth of the biofilm coverage varied greatly over the whole surface and its thickness reached $100 \mu \mathrm{m}$ in places (see Fig 3 ).

\section{Discussion}

In this study we have evaluated the effectiveness of disinfection/sterilisation procedures which are or have been in common use in dental practice to decontaminate syringes. We thought that the dental syringes, which are cannulated to allow passage of a disposable needle into an anaesthetic cartridge, might pose problems for penetration by disinfectant solutions and for steam during autoclaving.

Regulatory authorities such as the Australian Therapeutic Goods Administration (TGA) and the USA Food and Drug Administration (FDA) require in vitro testing of disinfectants and sterilants against various test organisms prior to registration. Organic material can decrease anti-microbial action of disinfectants both by inactivating the disinfectant and by physically protecting the microorganisms by preventing disinfectant access. For this reason disinfectant anti-microbial efficacy tests must be conducted in the presence of 'soil. ${ }^{14}$ The most common soil used for high grade disinfectant testing is $5 \%$ serum. However, for testing blood borne viruses the TGA now recommends $50 \%$ whole blood for disinfectant/sterilant efficacy testing ${ }^{13}$ due to the problem of whole blood inactivating disinfectants. We confirmed the ability of steam sterilisation and glutaraldehyde disinfection to inactivate DHBV in the presence of large amounts of soil in vitro.

In the second experiment virus was suspended in a blood/PBS mixture to simulate a blood and saliva mixture and used to contaminate dental syringes. All visible blood was removed from the dental syringes. No blood was evident inside the cannula on visual inspection. All ducklings in the control group became infected indicating that the bore of the needle inserted via the enclosed lumen of the contaminated dental syringe encountered sufficient numbers of DHBV to initiate infection. Despite removal of all visible blood $100 \%$ infection rate was also observed when syringes were washed and brushed in water.

Disinfectant manufacturers recommend flushing of holes and crevices with disinfectant before disinfection. Since the mechanical cleaning of instrument lumens is time consuming and potentially dangerous for the operator mechanical cleaning systems that reduce direct handling of contaminated instruments have gained acceptance. Ultrasonic cleaning baths are widely used in dental clinics and they have been shown to remove dried blood and saliva. ${ }^{16}$ Despite this sonication is ineffective in entering the lumen of fine tubing, and for that reason is not recommended for cleaning of cannulated instruments. ${ }^{5,17}$ The risk regarding ultrasonic treatment even in the presence of a detergent, as a substitute for sterilisation or even disinfection is evident from our results. Based on titration of DHBV in baby ducks the reduction in infectivity would represent less than a log decrease in viral titre (results not shown).

Following removal of visible blood the syringes were swirled around in the disinfectant to ensure even access. Soaking of contaminated syringes in $2 \%$ glutaraldehyde gave an unexpected result with most of the syringes capable of transmitting infection after 20minute contact time. The poor response obtained by glutaraldehyde is surprising as it has been shown to work efficiently in vitro on virus suspension in the presence of large amounts of blood and using short contact times (For instance see results of experiment 1). However, we $\mathrm{e}^{11}$ have previously shown that contact times of less than 5 minutes failed to completely inactivate DHBV present in blood contaminating the external surface of solid laparoscopes in vivo. We surmised that the increase in time required to kill DHBV was due to instrument-related factors. The longer inactivation time for DHBV in the laparoscope study and the failure of glutaraldehyde to decontaminate all the dental syringes compared with the in vitro results shown above warns against extrapolation of data obtained with suspension or carrier tests of test organisms to chemical disinfection of complex instruments.

In our study even autoclaving $\left(3\right.$ minutes at $\left.134^{\circ} \mathrm{C}\right)$ was associated with a significant failure rate with one of the sixteen ducks becoming infected. The recommended steam sterilisation times for the dental office are $15-20$ minutes at $121^{\circ} \mathrm{C}$ or 3 minutes at $134^{\circ} \mathrm{C}$. In practice, many surgeries have adopted the 3 minute cycle to facilitate high turnover of dental instruments, and have felt secure because of reported inactivation of microorganisms under these types of test conditions. The $\mathrm{BDA}^{5}$ recommend autoclaves with a pre-sterilisation vacuum phase for instruments that are wrapped, or are hollow or possess lumens. In contrast, in other countries ${ }^{3}$ downward displaced autoclaves are recommended for both wrapped (if a drying cycle is available) and unwrapped items. While in our in vitro test autoclaving a vial of blood:PBS for 3 minutes at $134^{\circ} \mathrm{C}$ completely inactivated the DHBV, transmission occurred following use of the processed unwrapped dental syringe despite removal of all visible blood. The survival of DHBV following high temperature, short cycle autoclaving is worrying as dentists commonly employ re-usable syringes.

In the electron microscopy study of dental syringes obtained from different Australian dental practices a biofilm deposit was evident in the lumen of all five dental syringes tested. We surmise that the gradual build up of biofilm with continual use would further compromise the ability to sterilise the syringe.

Bagging of instruments in suitable packaging material is recommended to ensure that the instruments reach the patients sterile. While the single instance of infection occurred with an unbagged syringe the number of tests performed was too small to provide a statistically valid comparison between the bagged and unbagged groups.

In practice longer autoclave cycles would permit greater heat conduction in any residual organic matter overlooked in cleaning, and this should be formally investigated to provide clear guidance for future regulatory guidelines. Additional cleaning regimes such as ultrasonic cleaning may enhance the effectiveness of heat sterilisation. Alternatively disposable syringes could be used as even the most efficient sterilisation can have a failure rate of $0.00001 \%{ }^{18}$

In summary, glutaraldehyde treatment and ultrasonication were not reliable treatment regimes for decontaminating dental syringes contaminated by duck hepatitis B virus. Our evidence suggests that longer autoclave cycles are required to ensure sterility of dental syringes and other instruments with complex narrow channels.

We would like to thank the staff of the Dental Clinic, Royal Prince Alfred Hospital for their valuable help and use of their autoclave. We also acknowledge the expert help and advice of Mr Tony Romeo of the Electron Microscope Unit, University of Sydney. Dr Karen Vickery was in receipt of a NHঊMRC Fellowship, Ms Aniko Pajkos was in receipt of a APAI Postgraduate Award. This research was partly funded by Laboratories Septodont, Paris, France and an ARC Collaborative Grant C49700121.

1 Chant K, Lowe D, Rubin G, et al. Patient-to-patient transmission of HIV in private surgical consulting rooms. The Lancet 1993; 342:1548-1549.

2 Robinson P, Challacombe S. Transmission of HIV in a dental practice the facts. Br Dent J 1993; 175(10):383-384.

3 NH\&MRC. Infection in the Health Care Setting: Guidelines for the prevention of transmission of infection. (1996) Pub: Aust. Government Printing Service, Canberra, Australia. 


\section{RESEARCH $\underline{\text { cross infection }}$}

4 Spaulding EH. Chemical disinfection of medical and surgical materials. In: Lawrence CA, Block SS (eds) Disinfection, sterilization and preservation. pp 517-31. Philadelphia:Lea and Febiger, 1968.

5 BDA Advisory Service. Infection control in dentistry. Advice sheet A12. pp. 3-23. January 2000.

6 Lowbury E J L, Ayliffe G A J, Geddes A M, Williams J D. Control of hospital infection. A practical Handbook. Pub London, Chapman and Hall. 1975.

7 Gibson G B, Mathias R G and Epstein J B. Compliance to recommended infection control procedures: changes over six years among British Columbia dentists. J Canadian Dent Ass 1995; 61:526-32.

8 Allen A, Organ R. Occult blood accumulation under the fingernails: a mechanism for the spread of blood borne infection. JADA 1982; 105:455459.

9 Bond W W, Favero M S, Petersen N J, Ebert J W. Inactivation of hepatitis B virus by intermediate-to-high level disinfectant chemicals. J Clin Microbiol 1983; 18:535-538.

10 Abebe A, Johansson B, Abens J, Strannegard O. Detection of enteroviruses in faeces by polymerase chain reaction. Scand J Infect Dis 1992; 24:265273.

11 Deva K, Vickery K, Zou J, West H, Harris J P, Cossart Y E. Establishment of an in use testing method for evaluating disinfection of surgical instruments utilising the duck hepatitis B model. Journal of Hospital Infection 1996; 33: 119-130.

12 Murray S M, Freiman J S, Vickery K, Lim D, Cossart Y C, Whiteley R K. Duck hepatitis B virus: a model to assess efficacy of disinfectants against hepadnavirus infectivity. Epidemiol Infect 1991;106, 435-443.

13 Prince D L, Prince N, Thraenhart O, Muchmore E, Bonder E, Pugh J. Methological approaches to disinfection of human hepatitis B virus. J Clin Microbiol 1993; 31:3296-3304.

14 Therapeutic Goods Administration Guidelines for evaluation of sterilants and disinfectants TGO\#54 Attachment 1. Pub Aust Government Printing Service, Canberra, Australia. 1997.

15 Freiman J S and Cossart Y E. Natural hepatitis B virus infection in Australia. Aust J Exp Biol Med Sci 1986; 64: 477-484.

16 Miller C H. Infection Control. Dent Clin Nth Am 1996;40:437-456.

17 ANCA Australian National Council on Aids. Infection control in office practices: Medical, dental and allied health. (1994). Australian Government Publishing Service, Canberra, Australia.

18 Miller C H. Sterilisation Disciplined Microbial Control. Dent Clin Nth Am 1991;35:339-355. 